\title{
High resolution computed tomography as a predictor of lung histology in systemic sclerosis
}

\author{
A U Wells, D M Hansell, B Corrin, N K Harrison, P Goldstraw, C M Black, R M du Bois
}

\begin{abstract}
Background The relative proportions of fibrosis and inflammation seen by open lung biopsy examination is a predictor of disease outcome in fibrosing alveolitis. This study was designed to assess the ability of high resolution computed tomography to predict the histological appearance of open lung biopsy specimens from patients with systemic sclerosis.
\end{abstract}

Methods Twenty abnormal biopsy specimens from 12 patients were assessed; abnormalities were categorised as fibrotic (fibrosis exceeding inflammation) or inflammatory (inflammation equal to or exceeding fibrosis). Computed tomography appearances were scored for the lobe from which the biopsy specimen was taken; scans were graded from parenchymal opacification alone through to a reticular pattern alone.

Results Two lobar appearances were identified on computed tomograms: amorphous parenchymal opacification equal in extent to reticulation (grade 3) and a predominantly reticular pattern (grade 4). There was a significant association between a fibrotic histological appearance and a grade 4 computed tomogram, and between an inflammatory histological appearance and a grade 3 computed tomogram. Computed tomography grade 4 was associated with a fibrotic histological appearance in 12 out of 13 lobes, and grade 3 with an inflammatory histological appearance in four out of seven lobes.

Conclusion Computed tomography discriminated between biopsy specimens that were predominantly fibrotic and a smaller group with a larger amount of inflammation.

Lung Institute, Roy

Heart and Lung

Hospital, London SW3

6NP

A U Wells

D M Hansell

B Corrin

N K Harrison

P Goldstraw

R M du Bois

Royal Free Hospital, London NW3 2QG

C M Black

Reprint requests to:

Dr R M du Bois, Interstitia Lung Disease Unit,

Emmanuel Kaye Building,

Royal Brompton National

Heart and Lung Hospital,

London SW3 6LR

Accepted 1 March 1992

Lung disease is common in systemic scler and is the major cause of death. ${ }^{1}$ The histological appearance of the lung is identical to that in cryptogenic fibrosing alveolitis. ${ }^{2}$ In cryptogenic fibrosing alveolitis the appearances on lung biopsy specimens provide prognostic information, a cellular specimen predicting a good response to treatment. ${ }^{3-5}$ The histological findings should therefore influence the therapeutic approach. Open lung biopsy by minithoracotomy has a high diagnostic yield and minimal morbidity. ${ }^{6}$ This procedure, however, cannot always be under- taken, and it cannot be repeated to monitor change.

High resolution computed tomography has an established role in the evaluation of interstitial lung disease. ${ }^{78}$ It is better than plain radiography in predicting a histological diagnosis of cryptogenic fibrosing alveolitis, lymphangiitis carcinomatosa, and sarcoidosis ${ }^{9}$ and more sensitive than plain radiography in detecting pulmonary abnormalities in systemic sclerosis. ${ }^{10}$ In cryptogenic fibrosing alveolitis the characteristic peripheral rim of reticular density corresponds histologically to areas of irregular fibrosis in open lung biopsy material ${ }^{11}$ and intra-alveolar and interstitial cellularity is seen as an amorphous or diffuse increase in lung density. ${ }^{1213}$ The ability of computed tomography to predict the proportions of fibrosis and cellularity in lung biopsy specimens has not been evaluated, particularly when parenchymal opacification and reticular abnormalities coexist. The distinction is important because the therapeutic response appears to depend on the relative extent of fibrosis and inflammation. ${ }^{5}$ If computed tomography alone is to guide treatment the technique must be able to determine which process predominates. This study was designed to assess whether computed tomography could achieve this aim.

\section{Methods}

PATIENTS

Twelve patients fulfilling the American Rheumatism Association preliminary criteria for the diagnosis of systemic sclerosis ${ }^{14}$ were assessed (10 female, 2 male; age (SD) 47.6 $(11.8)$ years). All underwent computed tomography less than six months (mean (SD) $2 \cdot 1(1 \cdot 5)$ months) before open lung biopsy. Twenty four lobes were biopsied via minithoracotomy; tissue was taken from a lower lobe and from the ipsilateral middle or upper lobe. ${ }^{6}$ The decision to perform open lung biopsy was made for clinical reasons with the fully informed consent of the patient; it was not part of a research protocol.

\section{HISTOLOGICAL GRADING} specimen were examined. Each of 24 biopsy specimens was scored semiquantitatively $(0-3)$ by a single observer (BC) for intra-alveolar inflammation, interstitial inflammation, interstitial fibrosis, and loss of alveolar architecture. The scores of the first two categories were added to give an "inflammation score" and the
All fields in all sections of each lung biopsy 
scores of the latter two to give a "fibrosis score."2 From these scores a final qualitative grading was derived as follows: (1) inflammation alone; (2) inflammation score exceeding fibrosis score; (3) inflammation score equal to fibrosisscore;(4)fibrosis scoreexceeding inflammation score; (5) fibrosis alone.

Grades 1, 2, and 3 were designated as inflammatory biopsy specimens and grades 4 and 5 as fibrotic (figs 1 and 2).

\section{HIGH RESOLUTION COMPUTED TOMOGRAPHY}

All computed tomography was performed on an Elscint 2002 scanner with a scanning time of 5.5 seconds. A high resolution protocol was performed with the window levels set for optimal imaging of the lungs (level -500 to 700 , width -1300 to -1600 Hounsfield units). The appearances of all computed tomography sections through the biopsied lobes were assessed by a single observer (DMH) without knowledge of the histological findings. A grading system was constructed on the basis of the method and observations of Muller et al, ${ }^{112}$ incorporating parenchymalopacification(seefig 3) and reticular abnormalities (fig 4), as follows: (1) parenchymal opacification alone; (2) parenchymal opacification more extensive than a reticular pattern; (3) parenchymal opacification equal in extent to a reticular pattern; (4) reticular pattern more extensive than parenchymal opacification; (5) reticular pattern alone.

One lobe was excluded from computed tomography evaluation because technical factors caused severe degradation of the image; the corresponding biopsy specimen was also excluded from the study.

\section{REPRODUCIBILITY OF GRADING SYSTEMS}

Intraobserver variability was assessed for histological and computed tomography grading systems. Histological appearances were assessed blind after an interval of one year by the same observer; 30 open lung biopsy specimens were graded, including three in which appearances were rated as normal on both occasions. In 18 of 27 abnormal biopsy specimens grading was identical on the two occasions; the remaining nine were graded consistently within one point on the five point scale. The most frequent disparity lay between grade 2 and grade 3; the distinction between "inflammatory" and "fibrotic" biopsy specimens (grades 1-3 versus grades 4 and 5) was made consistently in 25 of the 27 samples.

Computed tomography appearances were assessed blind after an interval of two months in 30 patients with systemic sclerosis; abnormal computed tomograms were selected at random from 65 patients with systemic sclerosis whose scans had been assessed on the first occasion. In 29 of the 30 computed tomography appearances were categorised as grade 3 or grade 4 at least once. Identical grades were assigned on the two occasions in 20 and in the remaining 10 the grades differed by only one point on the five point scale. The most frequent disparity lay between grade 2 and grade 3 ; the distinction between computed tomography grades $1-3$ and grades 4 and 5 was made consistently in 27 of the 30 samples.

\section{DATA ANALYSIS}

Computed tomography and histological gradings were compared for all abnormal biopsy specimens. The association between computed tomography and histological appearances was examined non-parametrically by means of the Fisher exact test.

\section{Results}

HISTOLOGY

Twenty three lung biopsy specimens were analysed. Three samples were normal; in each
Figure 1 Histological appearances of open lung biopsy specimen classified as inflammatory ("inflammation equal to fibrosis").

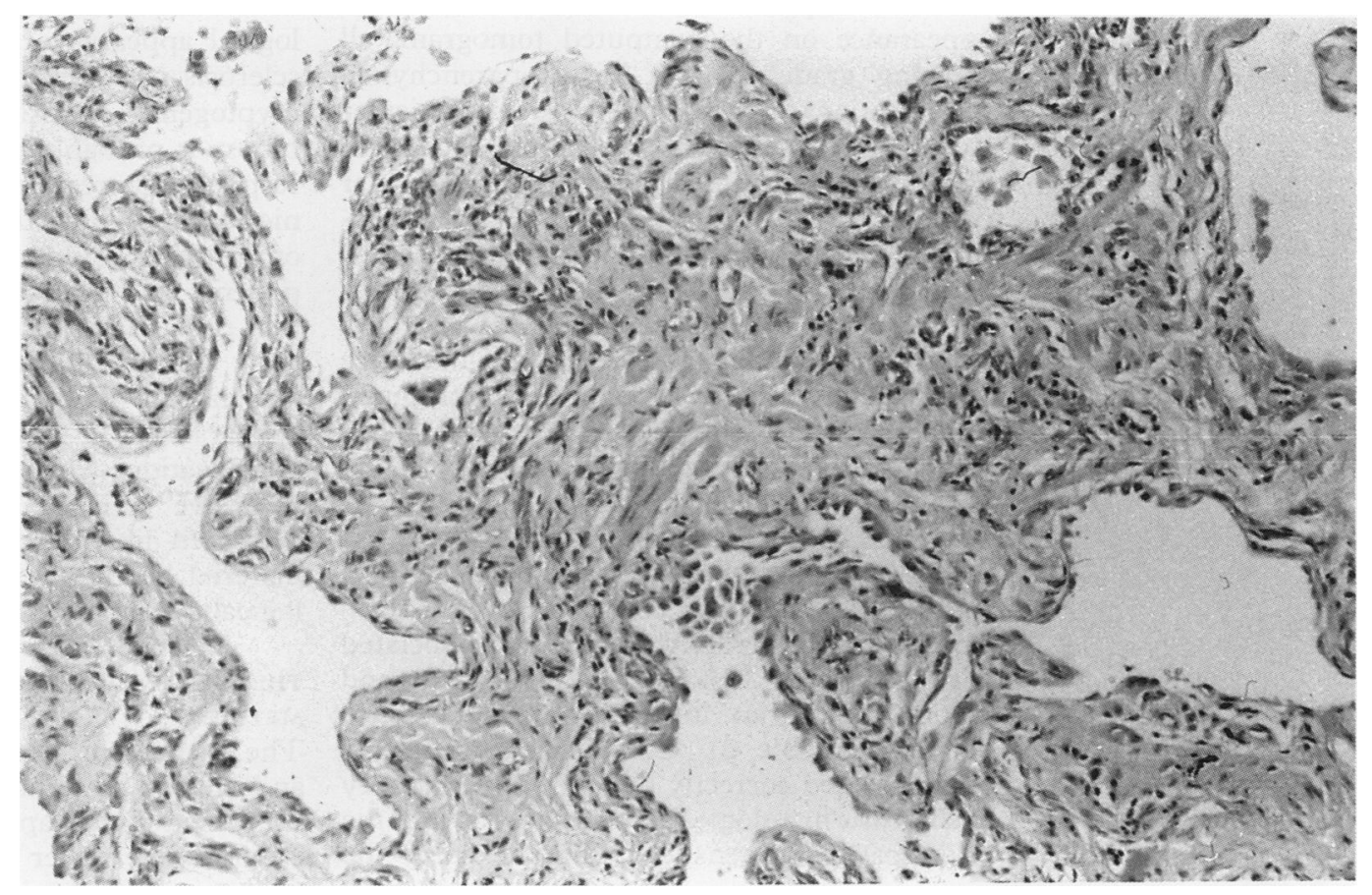


Figure 2 Histological appearances of open lung biopsy specimen classified as fibrotic ("fibrosis alone").

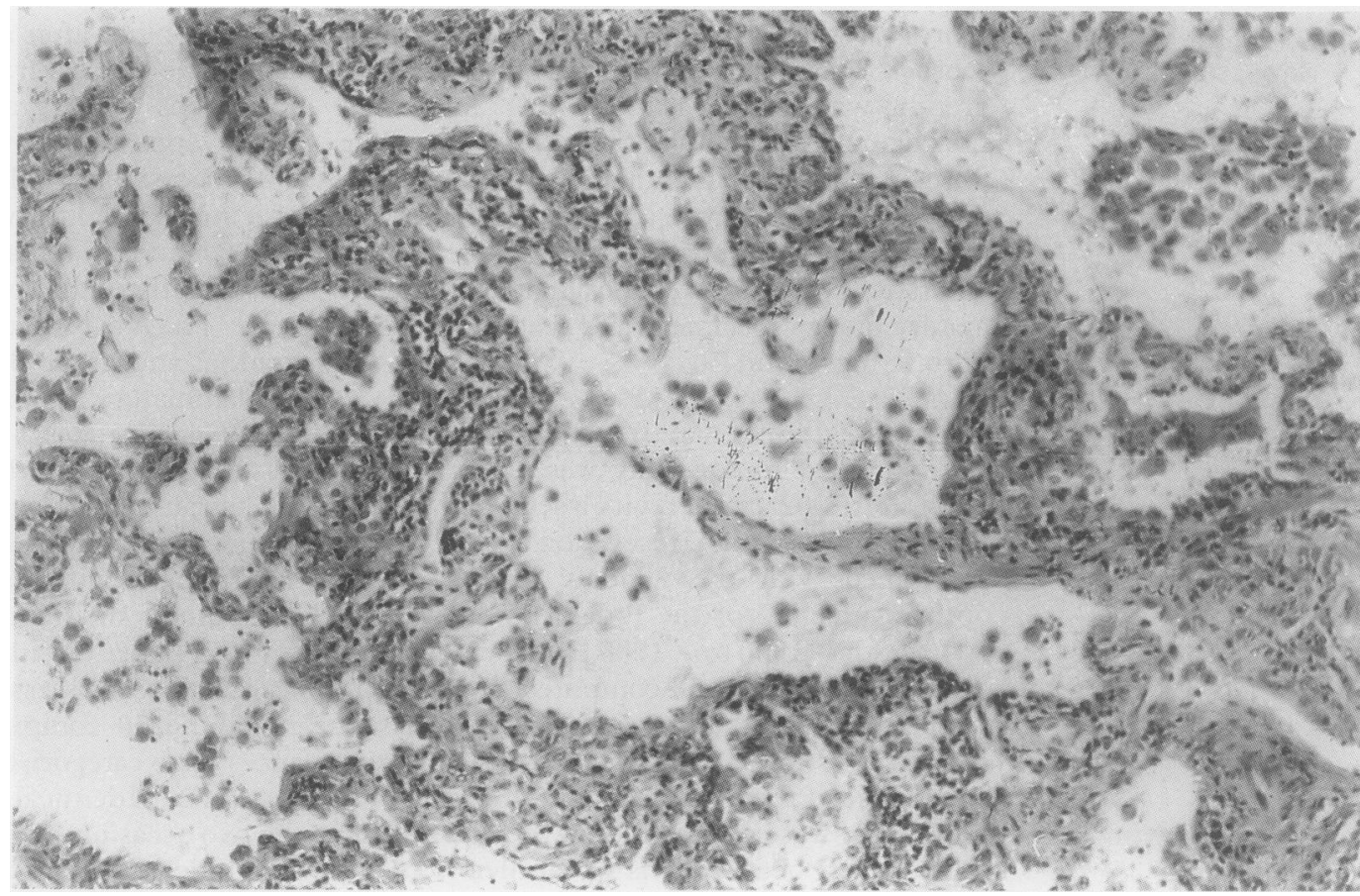

Table 1 The histological grades assigned to open lung biopsy specimens

\begin{tabular}{llr}
\hline Fibrotic specimens $(\mathrm{n}=15):$ & Grade 4 & 14 \\
Inflammatory specimens $(\mathrm{n}=5):$ & Grade 5 & 1 \\
& Grade 1 & 1 \\
& Grade 3 & 1 \\
Total biopsy specimens & & 3 \\
\hline
\end{tabular}

case a second biopsy specimen, taken from another site during the same operation, yielded abnormal tissue. There were five inflammatory (fig 1) and 15 fibrotic biopsy specimens (fig 2); histological gradings are detailed in table 1.

COMPUTED TOMOGRAPHY

All 23 biopsied lobes had an abnormal appearance on the computed tomogram, all showing grade 3 or 4 . In 10 parenchymal opacification (fig 3 ) was as extensive as reticular shadowing (the three normal biopsy specimens were in this group). In the other 13 a reticular pattern (fig 4) was the predominant abnormality. The lung biopsy appearances of the two computed tomography grades ( 3 and 4 ) have been compared in subsequent analyses. Analysis was confined to the 20 lobes that were adequately visualised on the computed tomograms and from which abnormal histological material was obtained.

ACCURACY OF COMPUTED TOMOGRAPHY IN DISCRIMINATING FIBROTIC AND INFLAMMATORY HISTOLOGICAL APPEARANCES

Computed tomography grade 4 was associated with a fibrotic histological appearance and grade 3 with an inflammatory appearance ( $<<0.04$; table 2). Computed tomography discriminated correctly between inflammatory and fibrotic histological appearances in 16 of the 20 biopsy specimens, including seven of the nine specimens obtained less than two months after computed tomography and nine of the 11 obtained two to six months after computed tomography.

Predominantly reticular abnormalities (computed tomography grade 4 ) were associated with a fibrotic appearance of the biopsy material in 12 of 13 lobes with this appearance. Computed tomography grade 3 was a less precise guide to histological appearance, but was associated with inflammatory histological appearance more frequently than was grade 4 ( $4 / 7$ versus $1 / 13 ; p<0.04)$.

\section{Discussion}

The aim of this study was to determine whether computed tomography could discriminate between a fibrotic and an inflammatory histological appearance in patients with systemic sclerosis who develop fibrosing alveolitis. In cryptogenic fibrosing alveolitis open lung biopsy is probably the best guide to the likely response to treatment. A non-invasive technique that predicts the histological appearances of the lungs would have obvious advantages for patients and clinicians. Our results indicate that predominantly reticular abnormalities on the computed tomogram are strongly associated with a fibrotic histological appearance. Amorphous parenchymal opacification when combined with equally extensive reticular shadowing identifies an increased likelihood of inflammatory biopsy material but is a less reliable guide to histological appearance than the reticular pattern.

\section{THE RELATIVE COMPUTED TOMOGRAPHY GRADING} METHOD and the two patterns of abnormality shown by computed tomography were each examined in isolation in earlier work. Biopsy specimens
The associations between histological findings 


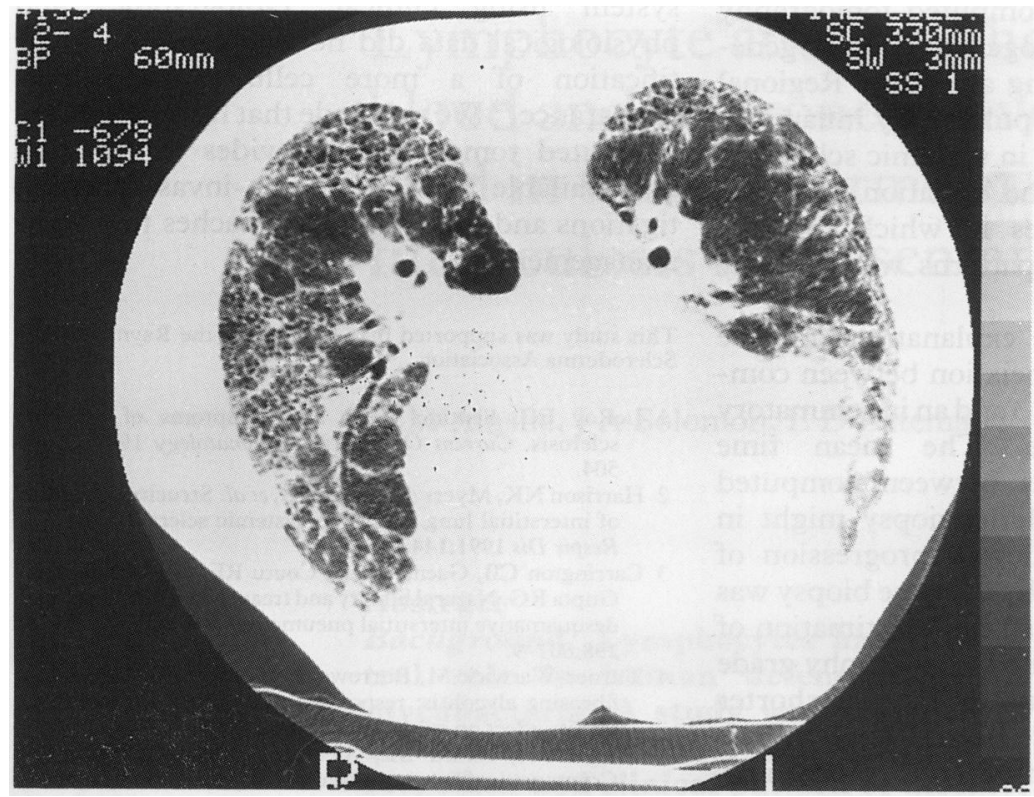

Figure 3 Amorphous parenchymal opacification on a high resolution computed tomogram from a patient with cryptogenic fibrosing alveolitis.

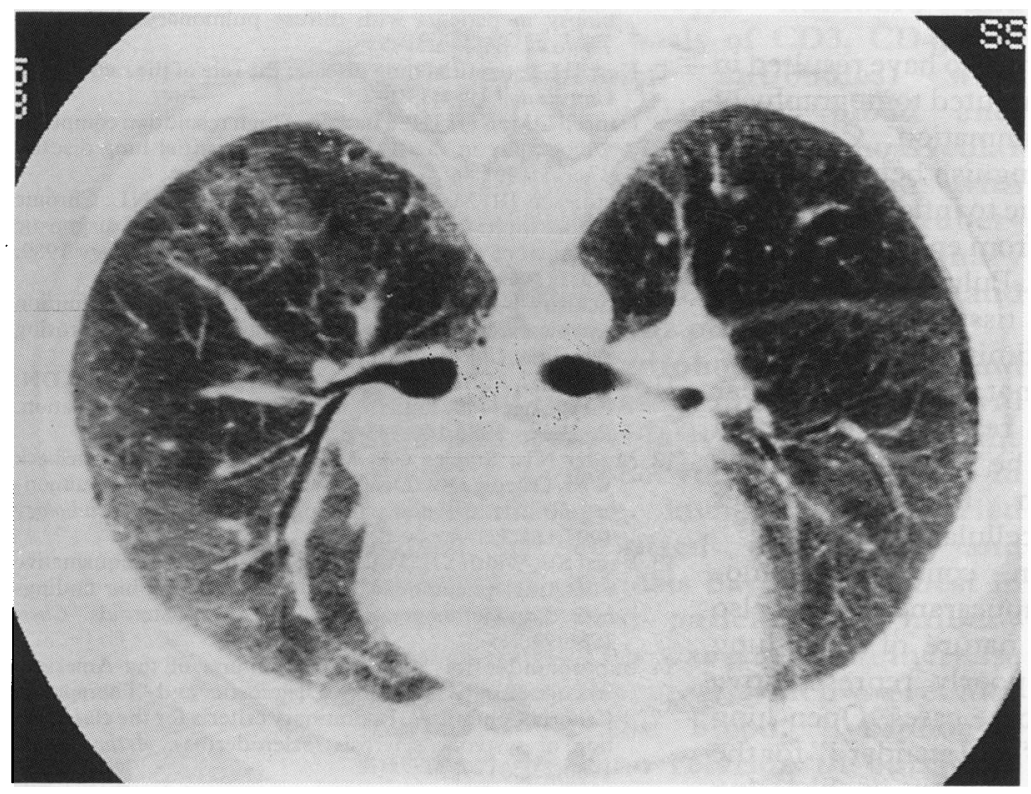

Figure 4 Extensive reticular abnormalities on a high resolution computed tomogram from a patient with cryptogenic fibrosing alveolitis.
Table 2 . Prevalence of inflammatory and fibrotic histological appearances in open lung biopsy specimens in relation to computed tomography $(C T)$ grades of the corresponding lobes-grade 3 (amorphous opacification equal in extent to reticulation) and grade 4 (predominant reticulation) *

\begin{tabular}{llc}
\hline & $\begin{array}{l}\text { Inflammatory } \\
\text { specimen } \\
(n=5)\end{array}$ & $\begin{array}{l}\text { Fibrotic } \\
\text { specimen } \\
(n=15)\end{array}$ \\
\hline CT grade $3(\mathrm{n}=7)$ & 4 & 3 \\
CT grade 4 $(\mathrm{n}=13)$ & 1 & 12 \\
\hline
\end{tabular}

^Association between computed tomography grade 4 and a fibrotic biopsy specimen and CT grade 3 and an inflammatory biopsy specimen: $p<0.04$.

relative grading system circumvents technical pitfalls in the estimation of absolute density on computed tomograms, as shown in studies of solitary pulmonary nodules in the last decade. ${ }^{15}$ It is difficult to standardise the measurement of tissue attenuation between computed tomography scanners. By comparison of increased parenchymal opacification with reticular abnormalities, without regard to an absolute measurement of either, this problem can be avoided.

ASSOCIATION BETWEEN COMPUTED TOMOGRAPHY AND HISTOLOGICAL APPEARANCES

A predominantly reticular pattern on the computed tomogram was strongly associated with a fibrotic histological picture, despite the fact that the computed tomography appearances of the whole lobe were compared with the regional histological appearances. The analysis of computed tomography appearances in the exact region of the biopsy would have been preferable, but was impracticable; $3 \mathrm{~mm}$ computed tomography sections are imaged at $10 \mathrm{~mm}$ intervals, precluding complete visualisation of the lungs. For this reason, and because the study was retrospective, it was decided to extend computed tomography analysis to the whole of the biopsied lobe. The strong association between computed tomography and histological appearances, despite this methodological limitation, suggests that predominantly reticular abnormalities on the computed tomogram identify diffuse fibrotic disease.

The presence of more extensive amorphous parenchymal opacification on computed tomograms identified lobes with a significantly higher prevalence of inflammatory histological appearances. The numbers in this subgroup were small, however, and there were relatively few patients with inflammatory appearances; this observation requires confirmation in a further study before it can be applied clinically with confidence. As fibrotic biopsy material was obtained from three of the seven biopsies, computed tomography grade 3 is not a precise guide to histological appearance.

This finding differs from earlier reports of a strong association between amorphous parenchymal opacification and inflammatory biopsy material ${ }^{12}$; in those patients, however, reticular abnormalities on the computed tomogram were not documented and may not have been present in some instances. In all the lobes assessed in directly. No attempt was made to derive absolute density scores for either pattern. A 
the present study the computed tomography appearances were heterogeneous, as is generally the case in fibrosing alveolitis. Regional histological variation in pulmonary inflammation is well documented in systemic sclerosis ${ }^{16}$ and may account for the variation in histological findings in lobes in which the two computed tomography patterns were equally extensive.

There are alternative explanations for the inconsistency of the association between computed tomography grade 3 and an inflammatory histological appearance. The mean time interval of two months between computed tomography and open lung biopsy might in some instances have allowed progression of inflammation to fibrosis by the time biopsy was performed, resulting in an underestimation of the ability of this computed tomography grade to predict histological appearance. A shorter time interval would have been desirable. The fact that computed tomography predictions were equally good whether the time interval to biopsy was less than two months or longer suggests that this was unlikely to have been a major source of inaccuracy.

Technical factors might also have resulted in an overestimation by computed tomography of the prevalence of inflammation. Computed tomography cannot distinguish between thickening of alveolar walls due to inflammatory cell infiltration and oedema from enhanced deposition of connective tissue. Pulmonary markings are superimposed when tissue dimensions lie outside the resolution limits of the scanner. Very fine fibrosis would not produce a reticular pattern, even with high resolution computed tomography, but would be "averaged" by the scanner, resulting in the appearances attributed by Muller to cellular infiltration. ${ }^{12}$

Discordance between computed tomography and histological appearances might also be due to the regional nature of open lung biopsy, resulting in a poorly representative histological sample in some cases. Open lung biopsy is the traditional "gold standard" for the assessment of disease activity in fibrosing alveolitis, although computed tomography "samples" more extensive areas of lung, including central regions that are not available to the surgeon.

Despite methodological and technical limitations, all of which might have reduced the strength of our observations, computed tomography identified a group of biopsy specimens in which predominant fibrosis was almost invariable and a further group with a higher prevalence of inflammation. This distinction has not been made in fibrosing alveolitis by other widely available noninvasive procedures. The chest radiograph is unreliable in showing whether alveolitis or fibrosis predominates. ${ }^{17} 18$ Pulmonary function tests, especially transfer factor for carbon monoxide, are sensitive indices of disease $\mathrm{e}^{19}{ }^{20}$ but do not distinguish between fibrosis and inflammation in lung interstitium. ${ }^{21} \mathrm{~A}$ composite scoring system using clinical, radiographic, and physiological data did not facilitate the identification of a more cellular histological appearance. ${ }^{22}$ We conclude that high resolution computed tomography provides information not available from other non-invasive investigations and may guide approaches to disease management.

This study was supported by a grant from the Raynaud's and Scleroderma Association.

1 LeRoy EC. Sentinel signs and symptoms of systemic sclerosis. Current Opinion in Rheumatology 1989;1:499504.

2 Harrison NK, Myers AR, Corrin B, et al. Structural features of interstitial lung disease in systemic sclerosis. Am Rev Respir Dis 1991;144:706-13.

3 Carrington CB, Gaensler EA, Coutu RE, FitzGerald MX, Gupta RG. Natural history and treated course of usual and desquamative interstitial pneumonia. N Engl J Med 1978; 298:801-9.

4 Turner-Warwick M, Burrows B, Johnson A. Cryptogenic fibrosing alveolitis: response to corticosteroid treatment and its effect on survival. Thorax 1980;35:593-9.

5 Wright PH, Heard BE, Steel SJ, Turner-Warwick M. Cryptogenic fibrosing alveolitis: assessment by graded ultrafine lung biopsy, histology compared with clinical, radiographic and physiological features. $\mathrm{Br} J$ Dis Chest 1981;75:61-70.

6 Venn GE, Kay PH, Midwood CJ, Goldstraw P. Open lung biopsy in patients with diffuse pulmonary shadowing. Thorax 1985;40:931-5.

7 Kerr IH. Interstitial lung disease: the role of the radiologist. Clin Radiol 1984;35:1-7.

8 Hansell DM, Kerr IH. The role of high resolution computed tomography in the diagnosis of interstitial lung disease. Thorax 1991;46:77-84.

9 Mathieson JR, Mayo JR, Staples CA, Muller NL. Chronic diffuse infiltrative lung disease: comparison of diagnostic accuracy of CT and chest radiography. Radiology 1989; 171:111-6.

10 Strickland B, Strickland NH. The value of high definition narrow section computed tomography in fibrosing alveolitis. Clin Radiol 1988;39:589-94.

11 Muller NL, Miller RR, Webb WR, Evans KG, Ostrow DN Fibrosing alveolitis: CT-pathologic correlation. Radiology 1986;160:585-8.

12 Muller NL, Staples CA, Miller RR, Vedal S, Thurlbeck WM, Ostrow DN. Disease activity in idiopathic pulmonary fibrosis: CT and pathological correlation. Radiology 1987;165:731-4

13 Vedal SV, Welsh VE, Miller RR, Muller NL. Desquamative interstitial pneumonia; computed tomographic findings before and after treatment with corticosteroids. Chest 1988;93:215-7.

14 Subcommittee for Scleroderma Criteria of the American Rheumatism Association Diagnostic and Therapeutic Criteria Committee. Preliminary criteria for the classification of systemic sclerosis (scleroderma). Arthr Rheum 1980;23:581-90.

15 Godwin JD. The solitary pulmonary nodule. Radiol Clin North Am 1983;21:709-21.

16 Harrison NK, Glanville AR, Strickland B, et al. Pulmonary involvement in systemic sclerosis: the detection of early changes by thin section CT scan, bronchoalveolar lavage and 99mTc-DTPA clearance. Respir Med 1989;83: 403-14.

17 Turner-Warwick M, Burrows B, Johnson A. Cryptogenic fibrosing alveolitis: clinical features and their influence on survival. Thorax 1980;35:171-80.

18 Hance AJ, Crystal RG. Idiopathic pulmonary fibrosis. In: Flenley DC, Petty TL Recent advances in respiratory medicine. Edinburgh: Churchill Livingstone, 1983: 249-87.

19 Owens GR, Fino GJ, Herbert DL, et al. Pulmonary function in progressive systemic sclerosis: comparison of CREST
syndrome variant with diffuse scleroderma. Chest 1983; syndrome vari

20 Alton E, Turner-Warwick $M$. Lung involvement in scleroderma. In: Jayson MIV, Black CM, eds. Systemic sclerosis: scleroderma. Chichester: Lilley, 1988:181-205.

21 Chinet T, Jaubert F, Dusser D, Danel C, Chrétien J, Huchon G. Effects of inflammation and fibrosis on pulmonary function in diffuse lung fibrosis. Thorax 1990;45:675-8.

22 Watters LC, King TE, Schwarz MI, Waldron JA, Stanford RE, Cherniak RM. A clinical, radiographic and physiologic scoring system for the longitudinal assessment of patients with idiopathic pulmonary fibrosis. Am Rev Respir Dis 1986;133:97-103. 


\title{
CORRECTION
}

In the paper by Dr AU Wells and others (July 1992;47:508-12) figure 1 should be transposed with figure 2 and figure 3 with figure 4 . The complete article is reprinted in the correct form.

\section{High resolution computed tomography as a predictor of lung histology in systemic sclerosis}

\author{
A U Wells, D M Hansell, B Corrin, N K Harrison, P Goldstraw, C M Black, R M du Bois
}

\begin{abstract}
Background The relative proportions of fibrosis and inflammation seen by open lung biopsy examination is a predictor of disease outcome in fibrosing alveolitis. This study was designed to assess the ability of high resolution computed tomography to predict the histological appearance of open lung biopóy specimens from patients with systemic sclerosis.
\end{abstract}

Methods Twenty abnormal biopsy specimens from 12 patients were assessed; abnormalities were categorised as fibrotic (fibrosis exceeding inflammation) or inflammatory (inflammation equal to or exceeding fibrosis). Computed tomography appearances were scored for the lobe from which the biopsy specimen was taken; scans were graded from parenchymal opacification alone through to a reticular pattern alone.

Results Two lobar appearances were identified on computed tomograms: amorphous parenchymal opacification equal in extent to reticulation (grade 3 ) and a predominantly reticular pattern (grade 4). There was a significant association between a fibrotic histological appearance and a grade 4 computed tomogram, and between an inflammatory histological appearance and a grade 3 computed tomogram. Computed tomography grade 4 was associated with a fibrotic histological appearance in 12 out of 13 lobes, and grade 3 with an inflammatory histological appearance in four out of seven lobes.

Conclusion Computed tomography discriminated between biopsy specimens that were predominantly fibrotic and a smaller group with a larger amount of inflammation.

Lung Institute, Royal

Brompton National

Heart and Lung

Hospital, London SW3

6NP

A U Wells

D M Hansell

B Corrin

N K Harrison

P Goldstraw

$\mathrm{R} M$ du Bois

Royal Free Hospital, London NW3 2QG

C M Black

Reprint requests to:

Dr R M du Bois, Interstitial Lung Disease Unit,

Emmanuel Kaye Building,

Royal Brompton National

Heart and Lung Hospital,

London SW3 6LR

Accepted 1 March 1992

Lung disease is common in systemic sclerosis logical appearance of the lung is identical to that in cryptogenic fibrosing alveolitis. ${ }^{2}$ In cryptogenic fibrosing alveolitis the appearances on lung biopsy specimens provide prognostic information, a cellular specimen predicting a good response to treatment. ${ }^{3-5}$ The histological findings should therefore influence the therapeutic approach. Open lung biopsy by minithoracotomy has a high diagnostic yield and minimal morbidity. ${ }^{6}$ This procedure, however, cannot always be under- taken, and it cannot be repeated to monitor change.

High resolution computed tomography has an established role in the evaluation of interstitial lung disease. ${ }^{78}$ It is better than plain radiography in predicting a histological diagnosis of cryptogenic fibrosing alveolitis, lymphangiitis carcinomatosa, and sarcoidosis and more sensitive than plain radiography in detecting pulmonary abnormalities in systemic sclerosis. ${ }^{10}$ In cryptogenic fibrosing alveolitis the characteristic peripheral rim of reticular density corresponds histologically to areas of irregular fibrosis in open lung biopsy material $^{11}$ and intra-alveolar and interstitial cellularity is seen as an amorphous or diffuse increase in lung density. ${ }^{1213}$ The ability of computed tomography to predict the proportions of fibrosis and cellularity in lung biopsy specimens has not been evaluated, particularly when parenchymal opacification and reticular abnormalities coexist. The distinction is important because the therapeutic response appears to depend on the relative extent of fibrosis and inflammation. ${ }^{5}$ If computed tomography alone is to guide treatment the technique must be able to determine which process predominates. This study was designed to assess whether computed tomography could achieve this aim.

\section{Methods}

PATIENTS

Twelve patients fulfilling the American Rheumatism Association preliminary criteria for the diagnosis of systemic sclerosis ${ }^{14}$ were assessed (10 female, 2 male; age (SD) 47.6 $(11 \cdot 8)$ years). All underwent computed tomography less than six months (mean (SD) $2 \cdot 1$ (1.5) months) before open lung biopsy. Twenty four lobes were biopsied via minithoracotomy; tissue was taken from a lower lobe and from the ipsilateral middle or upper lobe. ${ }^{6}$ The decision to perform open lung biopsy was made for clinical reasons with the fully informed consent of the patient; it was not part of a research protocol.

\section{HISTOLOGICAL GRADING}

All fields in all sections of each lung biopsy specimen were examined. Each of 24 biopsy specimens was scored semiquantitatively $(0-3)$ by a single observer (BC) for intra-alveolar inflammation, interstitial inflammation, interstitial fibrosis, and loss of alveolar architecture. The scores of the first two categories were added to give an "inflammation score" and the 
scores of the latter two to give a "fibrosis score." From these scores a final qualitative grading was derived as follows: (1) inflammation alone; (2) inflammation score exceeding fibrosis score; (3) inflammation score equal to fibrosisscore; (4)fibrosis score exceeding inflammation score; (5) fibrosis alone.

Grades 1,2, and 3 were designated as inflammatory biopsy specimens and grades 4 and 5 as fibrotic (figs 1 and 2).

\section{HIGH RESOLUTION COMPUTED TOMOGRAPHY}

All computed tomography was performed on an Elscint 2002 scanner with a scanning time of 5.5 seconds. A high resolution protocol was performed with the window levels set for optimal imaging of the lungs (level -500 to 700 , width -1300 to -1600 Hounsfield units). The appearances of all computed tomography sections through the biopsied lobes were assessed by a single observer (DMH) without knowledge of the histological findings. A grading system was constructed on the basis of the method and observations of Muller et al, ${ }^{112}$ incorporating parenchymalopacification(see fig 3 ) and reticular abnormalities (fig 4), as follows: (1) parenchymal opacification alone; (2) parenchymal opacification more extensive than a reticular pattern; (3) parenchymal opacification equal in extent to a reticular pattern; (4) reticular pattern more extensive than parenchymal opacification; (5) reticular pattern alone.

One lobe was excluded from computed tomography evaluation because technical factors caused severe degradation of the image; the corresponding biopsy specimen was also excluded from the study.

\section{REPRODUCIBILITY OF GRADING SYSTEMS}

Intraobserver variability was assessed for histological and computed tomography grading systems. Histological appearances were assessed blind after an interval of one year by the same observer; 30 open lung biopsy specimens were graded, including three in which appearances were rated as normal on both occasions. In 18 of 27 abnormal biopsy specimens grading was identical on the two occasions; the remaining nine were graded consistently within one point on the five point scale. The most frequent disparity lay between grade 2 and grade 3; the distinction between "inflammatory" and "fibrotic" biopsy specimens (grades 1-3 versus grades 4 and 5) was made consistently in 25 of the 27 samples.

Computed tomography appearances were assessed blind after an interval of two months in 30 patients with systemic sclerosis; abnormal computed tomograms were selected at random from 65 patients with systemic sclerosis whose scans had been assessed on the first occasion. In 29 of the 30 computed tomography appearances were categorised as grade 3 or grade 4 at least once. Identical grades were assigned on the two occasions in 20 and in the remaining 10 the grades differed by only one point on the five point scale. The most frequent disparity lay between grade 2 and grade 3; the distinction between computed tomography grades $1-3$ and grades 4 and 5 was made consistently in 27 of the 30 samples.

\section{DATA ANALYSIS}

Computed tomography and histological gradings were compared for all abnormal biopsy specimens. The association between computed tomography and histological appearances was examined non-parametrically by means of the Fisher exact test.

\section{Results}

HISTOLOGY

Twenty three lung biopsy specimens were analysed. Three samples were normal; in each
Figure 1 Histological appearances of open lung biopsy specimen classified as inflammatory

("inflammation equal to fibrosis").

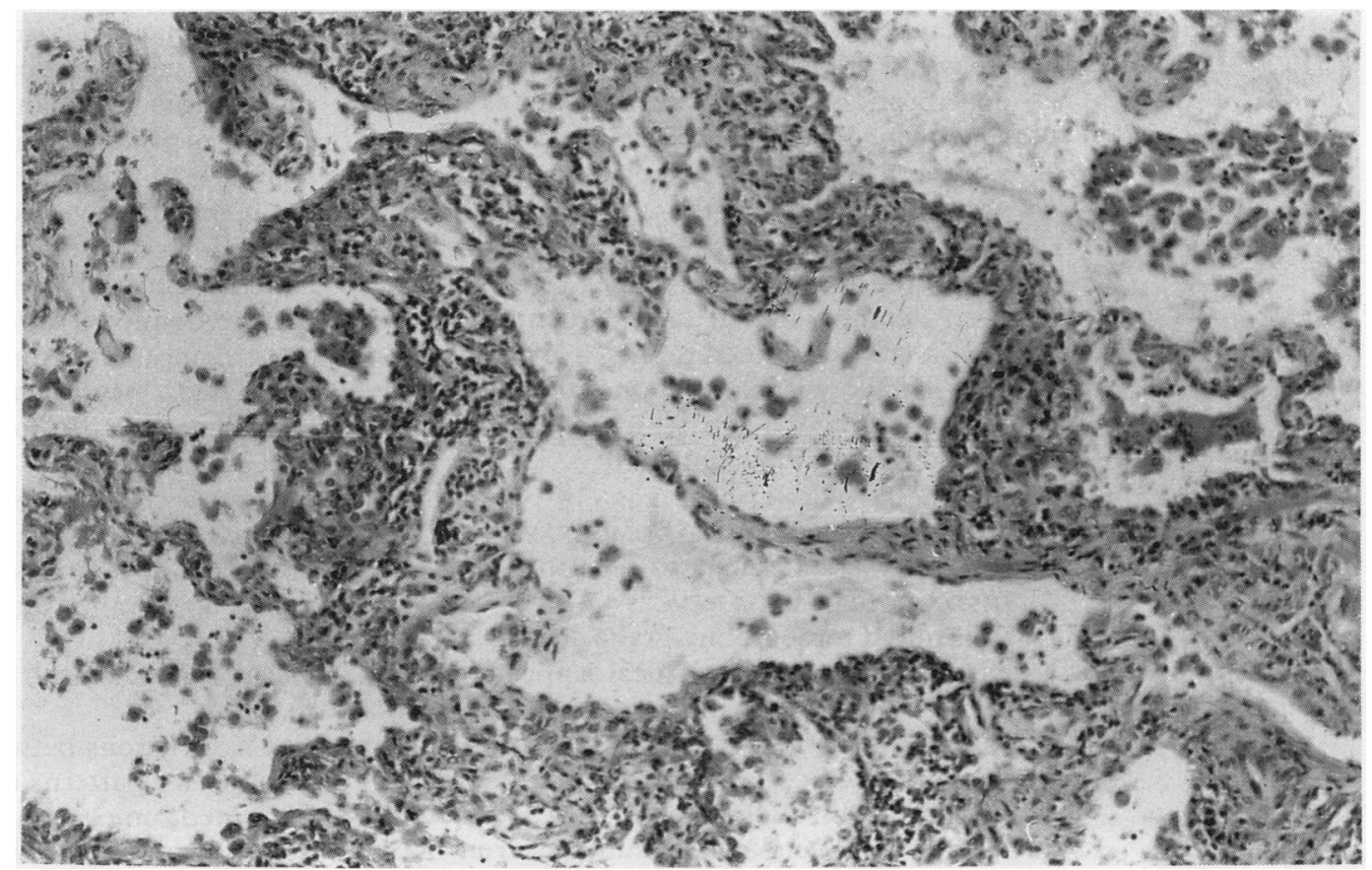


Figure 2 Histological appearances of open lung biopsy specimen classified as fibrotic ("fibrosis alone").

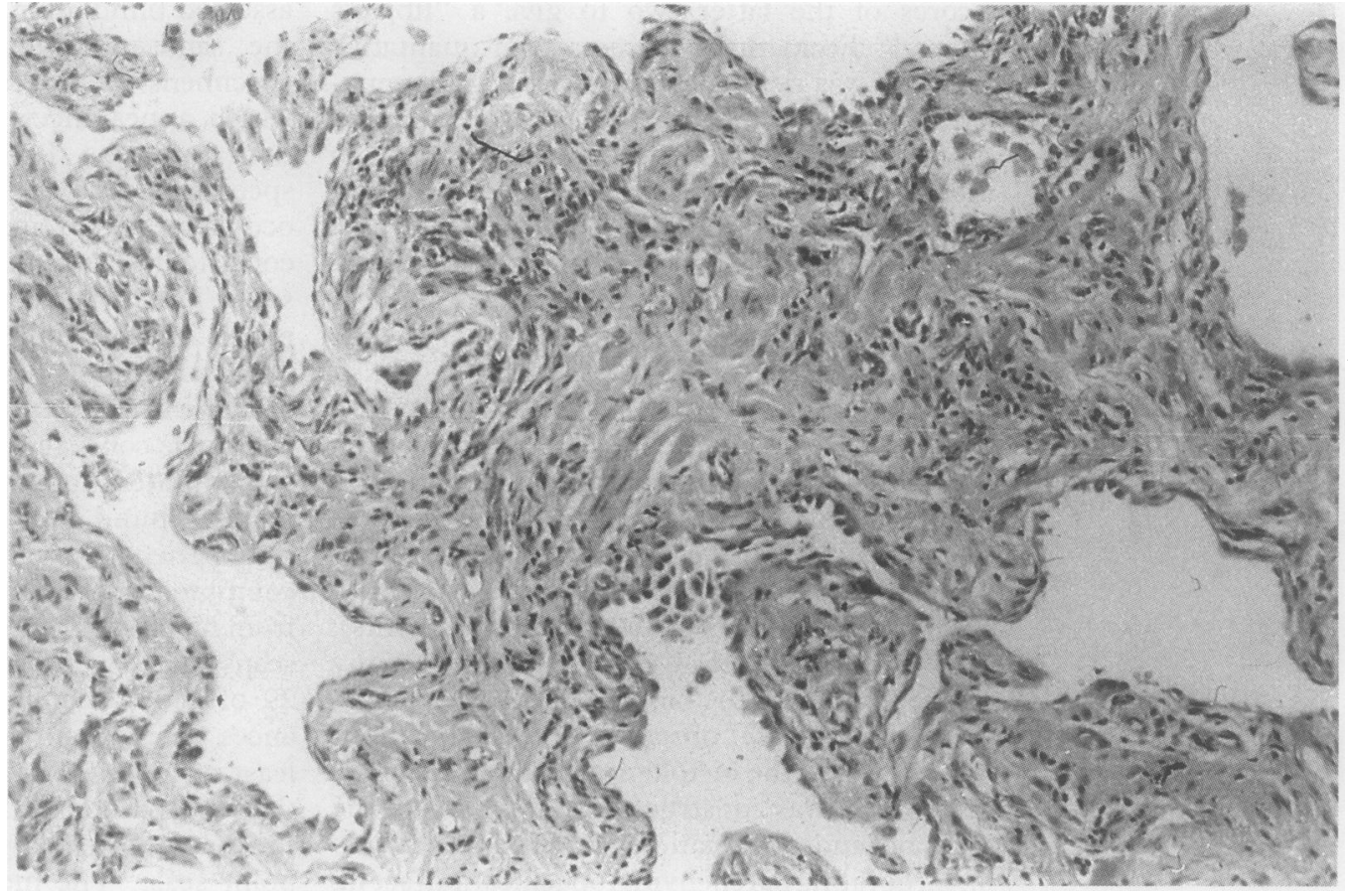

Table 1 The histological grades assigned to open lung biopsy specimens

\begin{tabular}{llr}
\hline Fibrotic specimens $(\mathrm{n}=15):$ & Grade 4 & 14 \\
& Grade 5 & 1 \\
Inflammatory specimens $(\mathrm{n}=5):$ & Grade 1 & 1 \\
& Grade 2 & 1 \\
& Grade 3 & 3 \\
Total biopsy specimens & & -20 \\
\hline
\end{tabular}

case a second biopsy specimen, taken from another site during the same operation, yielded abnormal tissue. There were five inflammatory (fig 1) and 15 fibrotic biopsy specimens (fig 2); histological gradings are detailed in table 1 .

COMPUTED TOMOGRAPHY

All 23 biopsied lobes had an abnormal appearance on the computed tomogram, all showing grade 3 or 4 . In 10 parenchymal opacification (fig 3 ) was as extensive as reticular shadowing (the three normal biopsy specimens were in this group). In the other 13 a reticular pattern (fig 4) was the predominant abnormality. The lung biopsy appearances of the two computed tomography grades ( 3 and 4 ) have been compared in subsequent analyses. Analysis was confined to the 20 lobes that were adequately visualised on the computed tomograms and from which abnormal histological material was obtained.

\section{ACCURACY OF COMPUTED TOMOGRAPHY IN} DISCRIMINATING FIBROTIC AND INFLAMMATORY HISTOLOGICAL APPEARANCES

Computed tomography grade 4 was associated with a fibrotic histological appearance and grade 3 with an inflammatory appearance ( $<<0.04$; table 2). Computed tomography discriminated correctly between inflammatory and fibrotic histological appearances in 16 of the 20 biopsy specimens, including seven of the nine specimens obtained less than two months after computed tomography and nine of the 11 obtained two to six months after computed tomography.

Predominantly reticular abnormalities ('computed tomography grade 4) were associated with a fibrotic appearance of the biopsy material in 12 of 13 lobes with this appearance. Computed tomography grade 3 was a less precise guide to histological appearance, but was associated with inflammatory histological appearance more frequently than was grade 4 (4/7 versus $1 / 13 ; p<0.04$ ).

\section{Discussion}

The aim of this study was to determine whether computed tomography could discriminate between a fibrotic and an inflammatory histological appearance in patients with systemic sclerosis who develop fibrosing alveolitis. In cryptogenic fibrosing alveolitis open lung biopsy is probably the best guide to the likely response to treatment. A non-invasive technique that predicts the histological appearances of the lungs would have obvious advantages for patients and clinicians. Our results indicate that predominantly reticular abnormalities on the computed tomogram are strongly associated with a fibrotic histological appearance. Amorphous parenchymal opacification when combined with equally extensive reticular shadowing identifies an increased likelihood of inflammatory biopsy material but is a less reliable guide to histological appearance than the reticular pattern.

\section{THE RELATIVE COMPUTED TOMOGRAPHY GRADING} METHOD

The associations between histological findings and the two patterns of abnormality shown by computed tomography were each examined in isolation in earlier work. Biopsy specimens 


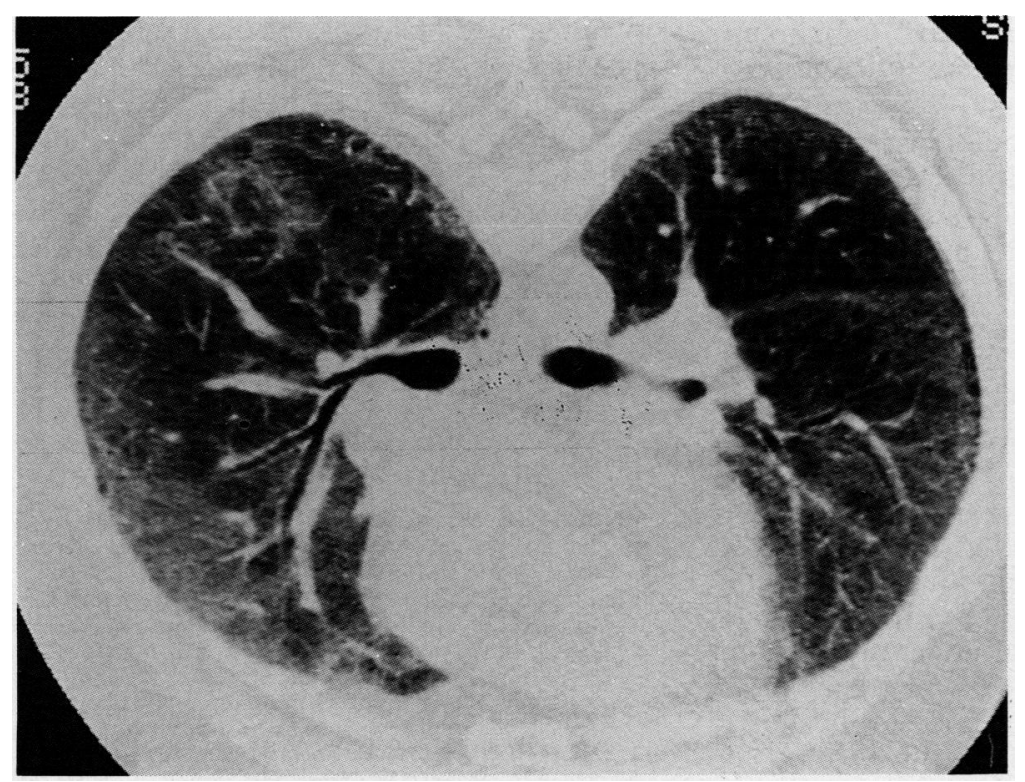

Figure 3 Amorphous parenchymal opacification on a high resolution computed tomogram from a patient with cryptogenic fibrosing alveolitis.

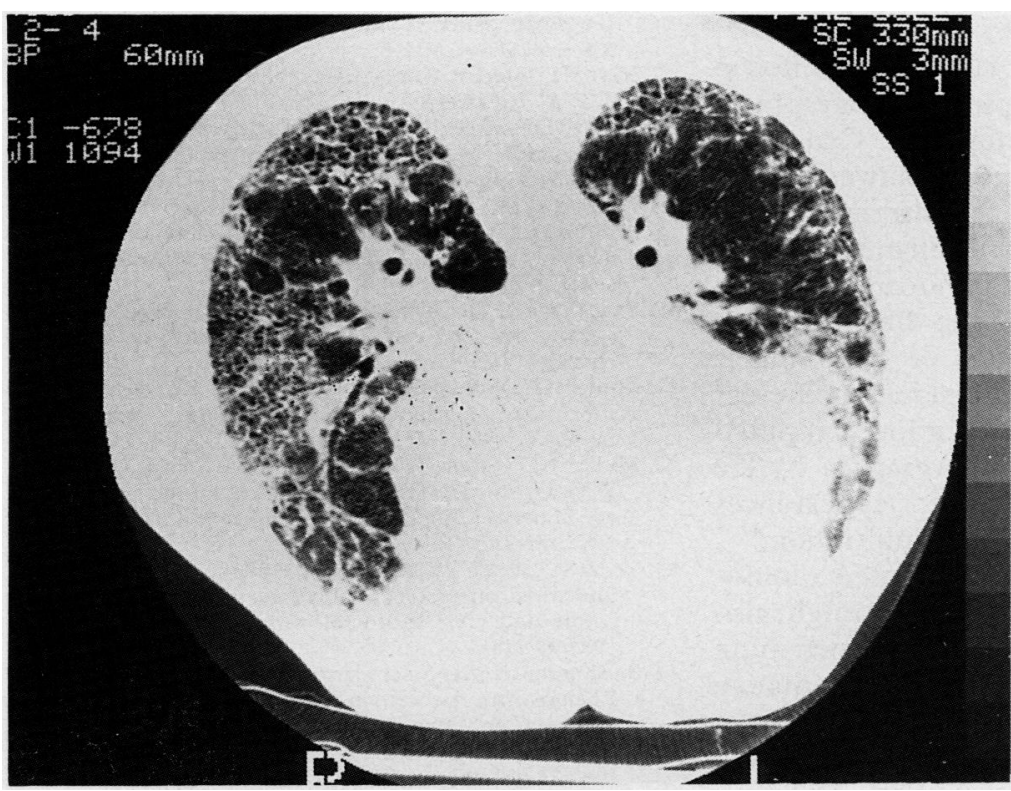

Figure 4 Extensive reticular abnormalities on a high resolution computed tomogram from a patient with cryptogenic fibrosing alveolitis.

from an area of peripheral reticulation on the computed tomogram are fibrotic whereas cellular biopsy material was associated with a "ground glass" appearance or an amorphous increase in lung density. ${ }^{1213}$ The computed tomography abnormalities described in these studies represent the ends of a spectrum, however. In fibrosing alveolitis computed tomography appearances generally consist of a mixture of parenchymal opacification and reticulation. If computed tomography is to be clinically useful in this context, it must discriminate accurately between inflammation and fibrosis when these abnormalities coexist on the computed tomogram.

For this reason the extents of the two computed tomogram abnormalities were compared directly. No attempt was made to derive absolute density scores for either pattern. A
Table 2 Prevalence of inflammatory and fibrotic histological appearances in open lung biopsy specimens in relation to computed tomography $(C T)$ grades of the corresponding lobes-grade 3 (amorphous opacification equal in extent to reticulation) and grade 4 (predominant reticulation) *

\begin{tabular}{llc}
\hline & $\begin{array}{l}\text { Inflammatory } \\
\text { specimen } \\
(n=5)\end{array}$ & $\begin{array}{l}\text { Fibrotic } \\
\text { specimen } \\
(n=15)\end{array}$ \\
\hline CT grade $3(\mathrm{n}=7)$ & 4 & 3 \\
CT grade 4 $(\mathrm{n}=13)$ & 1 & 12 \\
\hline
\end{tabular}

*Association between computed tomography grade 4 and a fibrotic biopsy specimen and CT grade 3 and an inflammatory biopsy specimen: $p<0.04$.

relative grading system circumvents technical pitfalls in the estimation of absolute density on computed tomograms, as shown in studies of solitary pulmonary nodules in the last decade. ${ }^{15}$ It is difficult to standardise the measurement of tissue attenuation between computed tomography scanners. By comparison of increased parenchymal opacification with reticular abnormalities, without regard to an absolute measurement of either, this problem can be avoided.

ASSOCIATION BETWEEN COMPUTED TOMOGRAPHY AND HISTOLOGICAL APPEARANCES

A predominantly reticular pattern on the computed tomogram was strongly associated with a fibrotic histological picture, despite the fact that the computed tomography appearances of the whole lobe were compared with the regional histological appearances. The analysis of computed tomography appearances in the exact region of the biopsy would have been preferable, but was impracticable; $3 \mathrm{~mm}$ computed tomography sections are imaged at $10 \mathrm{~mm}$ intervals, precluding complete visualisation of the lungs. For this reason, and because the study was retrospective, it was decided to extend computed tomography analysis to the whole of the biopsied lobe. The strong association between computed tomography and histological appearances, despite this methodological limitation, suggests that predominantly reticular abnormalities on the computed tomogram identify diffuse fibrotic disease.

The presence of more extensive amorphous parenchymal opacification on computed tomograms identified lobes with a significantly higher prevalence of inflammatory histological appearances. The numbers in this subgroup were small, however, and there were relatively few patients with inflammatory appearances; this observation requires confirmation in a further study before it can be applied clinically with confidence. As fibrotic biopsy material was obtained from three of the seven biopsies, computed tomography grade 3 is not a precise guide to histological appearance.

This finding differs from earlier reports of a strong association between amorphous parenchymal opacification and inflammatory biopsy material ${ }^{12}$; in those patients, however, reticular abnormalities on the computed tomogram were not documented and may not have been present in some instances. In all the lobes assessed in 
the present study the computed tomography appearances were heterogeneous, as is generally the case in fibrosing alveolitis. Regional histological variation in pulmonary inflammation is well documented in systemic sclerosis ${ }^{16}$ and may account for the variation in histological findings in lobes in which the two computed tomography patterns were equally extensive.

There are alternative explanations for the inconsistency of the association between computed tomography grade 3 and an inflammatory histological appearance. The mean time interval of two months between computed tomography and open lung biopsy might in some instances have allowed progression of inflammation to fibrosis by the time biopsy was performed, resulting in an underestimation of the ability of this computed tomography grade to predict histological appearance. A shorter time interval would have been desirable. The fact that computed tomography predictions were equally good whether the time interval to biopsy was less than two months or longer suggests that this was unlikely to have been a major source of inaccuracy.

Technical factors might also have resulted in an overestimation by computed tomography of the prevalence of inflammation. Computed tomography cannot distinguish between thickening of alveolar walls due to inflammatory cell infiltration and oedema from enhanced deposition of connective tissue. Pulmonary markings are superimposed when tissue dimensions lie outside the resolution limits of the scanner. Very fine fibrosis would not produce a reticular pattern, even with high resolution computed tomography, but would be "averaged" by the scanner, resulting in the appearances attributed by Muller to cellular infiltration. ${ }^{12}$

Discordance between computed tomography and histological appearances might also be due to the regional nature of open lung biopsy, resulting in a poorly representative histological sample in some cases. Open lung biopsy is the traditional "gold standard" for the assessment of disease activity in fibrosing alveolitis, although computed tomography "samples" more extensive areas of lung, including central regions that are not available to the surgeon.

Despite methodological and technical limitations, all of which might have reduced the strength of our observations, computed tomography identified a group of biopsy specimens in which predominant fibrosis was almost invariable and a further group with a higher prevalence of inflammation. This distinction has not been made in fibrosing alveolitis by other widely available noninvasive procedures. The chest radiograph is unreliable in showing whether alveolitis or fibrosis predominates. ${ }^{17} 18$ Pulmonary function tests, especially transfer factor for carbon monoxide, are sensitive indices of disease ${ }^{19} 20$ but do not distinguish between fibrosis and inflammation in lung interstitium. ${ }^{21} \mathrm{~A}$ composite scoring system using clinical, radiographic, and physiological data did not facilitate the identification of a more cellular histological appearance. ${ }^{22} \mathrm{We}$ conclude that high resolution computed tomography provides information not available from other non-invasive investigations and may guide approaches to disease management.

This study was supported by a grant from the Raynaud's and Scleroderma Association.

1 LeRoy EC. Sentinel signs and symptoms of systemic sclerosis. Current Opinion in Rheumatology 1989;1: 499-504.

2 Harrison NK, Myers AR, Corrin B, et al. Structural features of interstitial lung disease in systemic sclerosis. $A m R e v$ Respir Dis 1991;144:706-13.

3 Carrington CB, Gaensler EA, Coutu RE, FitzGerald MX, Gupta RG. Natural history and treated course of usual and desquamative interstitial pneumonia. N Engl J Med 1978; 298:801-9.

4 Turner-Warwick M, Burrows B, Johnson A. Cryptogenic fibrosing alveolitis: response to corticosteroid treatmen and its effect on survival. Thorax 1980;35:593-9.

5 Wright PH, Heard BE, Steel SJ, Turner-Warwick $M$ Cryptogenic fibrosing alveolitis: assessment by graded ultrafine lung biopsy, histology compared with clinical, radiographic and physiological features. $\mathrm{Br} J$ Dis Chest 1981;75:61-70.

6 Venn GE, Kay PH, Midwood CJ, Goldstraw P. Open lung biopsy in patients with diffuse pulmonary shadowing. Thorax 1985;40:931-5.

7 Kerr IH. Interstitial lung disease: the role of the radiologist. Clin Radiol 1984;35:1-7.

8 Hansell DM, Kerr IH. The role of high resolution computed tomography in the diagnosis of interstitial lung disease. Thorax 1991;46:77-84.

9 Mathieson JR, Mayo JR, Staples CA, Muller NL. Chronic diffuse infiltrative lung disease: comparison of diagnostic accuracy of CT and chest radiography. Radiology 1989; 171:111-6.

10 Strickland B, Strickland NH. The value of high definition narrow section computed tomography in fibrosing alveolitis. Clin Radiol 1988;39:589-94.

11 Muller NL, Miller RR, Webb WR, Evans KG, Ostrow DN Fibrosing alveolitis: CT-pathologic correlation. Radiology 1986;160:585-8.

12 Muller NL, Staples CA, Miller RR, Vedal S, Thurlbeck WM, Ostrow DN. Disease activity in idiopathic pulmonary fibrosis: CT and pathological correlation. Radiology 1987;165:731-4.

13 Vedal SV, Welsh VE, Miller RR, Muller NL. Desquamative interstitial pneumonia; computed tomographic findings before and after treatment with corticosteroids. Chest 1988;93:215-7.

14 Subcommittee for Scleroderma Criteria of the American Rheumatism Association Diagnostic and Therapeutic Criteria Committee. Preliminary criteria for the classification of systemic sclerosis (scleroderma). Arthr Rheum 1980;23:581-90.

15 Godwin JD. The solitary pulmonary nodule. Radiol Clin North Am 1983;21:709-21.

16 Harrison NK, Glanville AR, Strickland B, et al. Pulmonary involvement in systemic sclerosis: the detection of early changes by thin section CT scan, bronchoalveolar lavage and 99mTc-DTPA clearance. Respir Med 1989;83 403-14.

17 Turner-Warwick M, Burrows B, Johnson A. Cryptogenic fibrosing alveolitis: clinical features and their influence on survival. Thorax 1980;35:171-80.

18 Hance AJ, Crystal RG. Idiopathic pulmonary fibrosis. In Flenley DC, Petty TL Recent advances in respiratory medicine. Edinburgh: Churchill Livingstone, 1983 249-87.

19 Owens GR, Fino GJ, Herbert DL, et al. Pulmonary function in progressive systemic sclerosis: comparison of CREST syndrome variant with diffuse scleroderma. Chest 1983 . 84:546-50.

20 Alton E, Turner-Warwick $M$. Lung involvement in scleroderma. In: Jayson MIV, Black CM, eds. Systemic sclerosis: scleroderma. Chichester: Lilley, 1988:181-205.

21 Chinet T, Jaubert F, Dusser D, Danel C, Chretien J Huchon G. Effects of inflammation and fibrosis on pulmonary function in diffuse lung fibrosis. Thorax 1990;45:675-8.

22 Watters LC, King TE Schwarz MI, Waldron JA, Stanford RE Cheriak RM A clinical, radiographic and her physiologic scoring system for the longitudinal assessmen of patients with idiopathic pulmonary fibrosis. $A m R e v$ Respir Dis 1986;133:97-103. 